\title{
Kernos
}

Revue internationale et pluridisciplinaire de religion grecque antique

$10 \mid 1997$

Varia

\section{Expérimenter dans le champ des polythéismes}

\section{Marcel Detienne}

URL : http://journals.openedition.org/kernos/645

DOI : $10.4000 /$ kernos. 645

ISSN : 2034-7871

\section{Éditeur}

Centre international d'étude de la religion grecque antique

Édition imprimée

Date de publication : 1 janvier 1997

Pagination : $57-72$

ISSN : 0776-3824

Référence électronique

Marcel Detienne, «Expérimenter dans le champ des polythéismes », Kernos [En ligne], 10 | 1997, mis en ligne le 12 avril 2011, consulté le 01 mai 2019. URL : http://journals.openedition.org/kernos/645 ; DOI : 10.4000/kernos.645 


\section{Expérimenter dans le champ des polythéismes}

Que les dieux soient de bons objets n'est pas une découverte de l'anthropologie contemporaine. Les premiers anthropologues n'ont jamais oublié de prescrire l'inventaire des différents types de puissances déambulant dans un village ou distribués dans l'étendue d'un royaume. Dès la fin du XIX ${ }^{e}$ siècle, toutefois, deux convictions bien partagées ont fait obstacle à l'analyse de ces vastes populations de puissances surnaturelles, réparties entre les sociétés archaïques et les civilisations alors les plus respectées dans l'Histoire telles, au hasard, la Chine, l'Inde et la Grèce. Les premiers comparatistes entre Tylor et Frazer étaient, semble-t-il, persuadés que l'élément stable, sinon permanent, d'une culture à l'autre, était le rituel organisé autour d'un thème, avec grandes architectures festives et temps forts du calendrier. Ce qui justifiait leur attention si vive pour les scénarios des tournants de l'année ou les aventures de la royauté magique en Afrique, dans le monde Indien et dans les civilisations classiques entre l'Italie et la Scandinavie.

Par ailleurs, les mêmes anthropologues, convaincus que les entités divines passent, disparaissent et réapparaissent sans raison, s'accordaient volontiers à penser que chacune des figures de ce monde surnaturel si fluide et inconsistant devait être expliquée en soi dans la mesure où elle pouvait revendiquer un nom et quelque trait singulier. Tout au long du XIX ${ }^{e}$ siècle, l'ethnologie ainsi que l'histoire des religions s'inquiètent de genèse et d'évolution. Les représentations de l'âme, les différents types d'esprit, les formes d'entités supérieures, autant d'approches pertinentes pour le problème majeur : origine et développement de l'idée du divin, entre partisans de l'animisme, adeptes du totémisme et théoriciens des formes élémentaires de la vie religieuse. Dans le champ de la religion grecque, à l'écart des habitants de la Nigritie appelés par le Président de Brosses à témoigner de l'idolâtrie et du fétichisme des origines, le philosophe semble un précurseur: Hegel reconnaît l'existence d'un panthéon peuplé de dieux qui vivent ensemble et d'une vie personnelle, avec des passions et des intérêts opposés. Les dieux de l'Olympe cessent d'être de froides allégories, chacune sur son piédestal; chaque dieu devient une forme signifiante, mais le monde «polythéique » - polytbeos disent les Grecs - semble incapable de 
s'organiser en une totalité systématiquement articulée ${ }^{1}$. L'Olympe se découvre alors au miroir d'Homère et ceux qui en font l'histoire, entre Creuzer et Welcker, sont plus préoccupés de découvrir dans les dieux de la Grèce une intuition de l'absolu ${ }^{2}$ que les groupements différenciés observés par Pausanias dans sa description de la Grèce au temps d'Hadrien.

Dans les premières années du $x^{\mathrm{e}}$ siècle, l'écart se creuse entre « les dieux des carrefours », ceux de la Nigritie, et les grands dieux du polythéisme rendu à son état paradigmatique de naguère, c'est-à-dire confiné à la culture grecque fermée sur elle-même, et redevenue chasse gardée de ses historiens patentés, indifférents à toute réflexion comparative sur les ensembles polythéistes, hier et aujourd'hui. Entre les années vingt et les années quarante, le pré carré des dieux grecs voit s'affronter historiens positivistes et interprètes spiritualistes. D'un côté, l'érudition parfaite de Martin P. Nilsson qui interroge les puissances divines à partir du bon sens, et d'un solide fonds paysan, permettant d'identifier les représentations simples que le travail de l'imaginaire et de l'histoire va habiller de figures multiples et bariolées. De l'autre, l'herméneutique intelligente de Walter F. Otto, s'exerçant à découvrir comment chaque dieu signifie une sphère de l'existence, une expérience exemplaire de l'homme «présent au monde ». Deux approches qui ne sont pas sans vertus, mais qui partagent la même indifférence envers la nature même du polythéisme : un système complexe de relations entre puissances divines ou entités surnaturelles.

C'est avec et sous l'influence de Georges Dumézil que, dans les années soixante, des historiens et des anthropologues commencent à s'intéresser aux ensembles polythéistes eux-mêmes, aux modes d'organisation de ces systèmes de dieux ainsi qu'aux différentes manières dont des sociétés comme la Grèce, Rome et l'Inde se pensent à travers des entités religieuses. À l'approche historico-génétique qui régnait de son temps, Dumézil a substitué une analyse «structurelle » tournée vers les complémentarités, les oppositions, les hiérarchies et les complexes de relations. Après vingt ans d'analyses des configurations de dieux et des microsystèmes dispersés dans le champ indo-européen, son domaine d'élection, Georges Dumézil commence à exercer une influence majeure sur les travaux qui décident de prendre au sérieux les dieux, que ce soit dans le Caucase ${ }^{3}$, en Grèce ${ }^{4}$ ou dans l'Inde ${ }^{5}$.

\footnotetext{
$C f$. l'esquisse en forme de préface «Au commencement était le corps des dieux », écrite pour la version française de W. F. OTTO, Les dieux de la Grèce. La figure du divin au miroir de l'esprit grec (1929), Paris, 1984, p. 7-19.

2 B. Bravo, Dieu et les dieux chez F. Creuzer et F.G. Welcker, in Fr. SCHMIDT (éd.), L'impensable polytbéisme, Paris, 1988 , p. 375-424.

3 Cf. G. CHARACHIDZÉ, Le système religieux de la Géorgie païenne. Analyse structurelle d'une civilisation, Paris, 1968.

4 Cf. J.-P. VERNANT, Mythe et pensée chez les Grecs, Paris, 1965, p. 97-143 (= L'Homme, 1963).

5 Cf. L. DUMONT, Une sous-caste de l'Inde du Sud. Organisation sociale et religion des Pramalai Kallar, Paris, 1957, p. 396-403 (= Journal Asiatique, 1953).
} 
Il convient de s'y arrêter : les dieux que Dumézil découvre et fait découvrir ont joué un rôle essentiel dans l'étude comparative (et expérimentale) des «religions des peuples indo-européens ». Tel est à peu près l'intitulé de la direction d'études à l'École pratique des Hautes Études (Sciences Religieuses) où Dumézil va travailler pendant trente ans. L'ancienne «mythologie comparée », choisie en 1935, a disparu en 1945 , et, dès 1948 , elle va céder la place à la « civilisation indo-européenne », cette fois au Collège de France et dans un parcours parallèle ${ }^{6}$. L'entreprise intellectuelle de Dumézil commence avec des dieux articulés, des dieux en groupements, des assemblages de puissances divines. Ces dieux dont l'ancienne «mythologie comparée » voulait appréhender l'essence et l'étymologie sur la base d'équations linguistiques, le nouveau comparatisme, établi dans l'enceinte du monde indo-européen, privilégie en eux l'ordre de leur énumération, leurs relations hiérarchiques, les formes d'opposition et de complémentarité qui permettent d'explorer les données «théologiques» comme la triade précapitoline (Jupiter, Mars, Quirinus), la triade des dieux d'Upsal (Odhinn, Thôrr, Freyer), la liste des Âditya dans l'Inde ancienne, celle des Entités (des Amosa Spanta) du Zoroastrisme dans l'Iran ancienne ${ }^{7}$. Données complexes mais données primaires qui imposent ce que Dumézil appellera « le fait de la structure ${ }^{8}$, donnant accès à cette «ultra-histoire » de la civilisation indoeuropéenne qui va s'enrichir des ensembles articulés de concepts, détectés sur un vocabulaire commun très charpenté, formant l'armature d'un système de pensée doté d'« astérisques » et toujours proto- sinon préhistorique.

Des enquêtes de Dumézil, les analystes des ensembles polythéistes, sans contraintes indo-européennes, vont retenir un certain nombre de principes. Le premier que nous venons d'évoquer, c'est l'attention portée aux structures immédiates de tant de polythéismes : autels à dieux multiples, sanctuaires à plusieurs dieux, fêtes et rituels qui, tantôt, mettent en relation deux divinités réunies pour l'occasion, tantôt, associent deux aspects d'une même puissance, contrastés par des marques, sacrificielles par exemple. Les sociétés polythéistes, d'hier et d'aujourd'hui, sont riches en collections de divinités, en groupements circonstanciels ou récurrents, en configurations monumentales ou éphémères. Le déja-structurel (pour respecter le vocabulaire de Dumézil) est le matériau premier de l'analyste, convié à la cueillette avant de se mettre en chasse. Les « structures » sont là, il suffit de ne pas les piétiner, « pensées sauvages » qu'un peu d'habileté suffit à dégager avec, déjà, ce qu'elles signifient ou commencent à signifier.

Le deuxième principe qui découle du premier mais conduit ainsi à dépasser les limites du cadre trifonctionnel, si nécessaire aux avancées duméziliennes, c'est qu'un dieu ne peut se définir en termes statiques. À l'analyste de faire le

Cf. Problèmes et méthodes d'Histoire des Religions, Paris, 1968, p. 165-167.

7 Deux repères : G. DumÉzIL, Les dieux des Indo-Européens, Paris, 1952; Les dieux souverains des Indo-Européens, Paris, 1977.

8 Cf. e.g. G. DUMÉZIL, Mythe et Epopée, III. Histoires romaines, Paris, 1973, p. 10-16. 
relevé de l'ensemble des positions occupées par une divinité dans l'ensemble d'un système polythéiste. À côté de ce qui est dit « en clair »" des dieux et de leur action - et que le savoir d'une ultrahistoire entend privilégier dans chaque culture -, il faut repérer toutes les formes d'association et de contraste expérimentées ou simplement suggérées par une culture, jusque dans ses potentialités. Chemin faisant, l'analyste, convaincu qu'il doit étudier les dieux les uns par rapport aux autres, s'exercera à définir leurs limites respectives, à délimiter leurs champs d'action les uns par rapport aux autres. Le présupposé commun à tant de recherches neuves, faites ou autorisées par Georges Dumézil, c'était, il faut y insister, que la validation la plus sûre d'une analyse de relations entre dieux ou la définition d'un champ d'action propre à une puissance divine devait venir d'un énoncé indigène et, mieux encore, d'un énoncé de nature théologique. Produit par des théologiens polythéistes, souvent identiques aux « administrateurs de la mémoire » qui, de relais en relais, ont transmis le plus conscient avec le reste, abandonné à l'inconscient historique du langage et de la civilisation qu'il véhicule.

Le dernier principe, esquissé par Dumézil en 1949 et dessiné au trait en 1966, était à la fois le plus neuf et le plus malaisé à mettre en ouvre ${ }^{10}$. Le plus neuf parce qu'il introduisait une distinction forte entre le champ d'action d'un dieu et son mode d'action. Le champ d'action d'une puissance divine englobe les lieux et les occasions de ses services, et certaines d'entre elles, comme entraînées par l'hypertrophie de la «fonction » à laquelle elles sont rattachées, se trouvent amenées à intervenir dans des domaines fort éloignés de ce qui semble leur province première. Il ne faut donc pas regarder " où intervient un dieu, mais comment il intervient $»$. Définir le mode d'action d'un dieu, c'est donc au-delà des points d'application de son action, des décors où il paraît, chercher à atteindre « une manière et des moyens constants d'agir ». Le mode d'action doit être précis, spécifique, appartenant à un seul dieu. Principe très nouveau quand il s'ouvrait sur des pratiques expérimentales comme celles que Dumézil a imaginées très tôt, mais qui venait buter, dès sa mise en application, sur les figures porteuses de la civilisation indo-européenne à découvrir, c'est-à-dire sur les trois fonctions et l'appareil de la tripartition. Fonction, pour Dumézil, est synonyme d'activité, activité sociale, activités des hommes en société, ceux qui exercent le pouvoir, ceux qui font la guerre, ceux qui produisent les biens et les nourritures. Les dieux les plus importants, ceux qui président, seuls ou en couple, aux grandes fonctions, exercent donc des activités; ce sont des agents et très affairés. Dumézil les appelle souvent les «patrons », figures divines dont il analyse sans relâche les relations, d'une fonction à l'autre, et sans négliger les différents « aspects » d'une même fonction, mais en insistant plus volontiers sur

9 Gf. e.g. G. DUMÉZIL, Rituels indo-européens à Rome, Paris, 1954, p. 78.

10 G. DUMÉZLl, L'béritage indo-européen à Rome, Paris, 1949, p. 1; La religion romaine archaique, Paris, 1966, p. 179 et 229 
les caractères fondamentaux d'un dieu patron, tout en tenant à l'écart les services « latéraux et accessoires ».

Arrêtons-nous un instant sur la formule: « les caractères fondamentaux d'un dieu patron $»^{11}$. Elle fait écho à ce que les Grecs, entre Homère et Hérodote, pensent tout haut de leurs dieux : que chacune des puissances divines a reçu en partage une timé, un domaine de compétence qui est sa part d'honneur, domaine ayant la forme d'une limitation assignée par le partage. Le plus fameux est celui auquel procède le nouveau Roi des dieux après sa victoire sur les Titans. Un dieu, une déesse reçoivent donc ce que l'Iliade appelle des erga : des travaux, des cuvres, des activités. Dont les dieux et les hommes sont conscients quand ils en parlent ensemble. Au chant V de l'fliade, Aphrodite s'élance au secours d'Énée, en grand danger face à Diomède lequel reconnaît aussitôt Aphrodite et se réjouit de la mettre à mal, par la force. «Il sait qu'Aphrodite est une déesse sans force (analkis), qu'elle n'est pas de ces divinités qui président aux combats humains; elle n'est ni Athéna, ni Enyô qui dévaste les cités $\gg^{12}$. Aphrodite est immédiatement blessée, son sang, son ichôr, se met à couler; défaillante, elle se traîne jusqu'à son frère-amant, Arès, lui demandant instamment de l'évacuer. Et Zeus la console en lui tapotant la joue et lui rappelant que ce n'est pas à elle qu'ont été données les œuvres de guerre (polemèia erga) : "Consacre-toi, pour ta part, aux douces ouvres d'hyménée (erga gamoio) ». Deux « fonctions », deux domaines d'activité distincts, séparés, soigneusement délimités. En apparence, car il n'est étranger à aucun observateur du polythéisme grec que, « dans les douces œuvres d'hyménée », Aphrodite n'est pas seule; qu'il y a Héra dite l'Accomplie, teleia, en compagnie de Zeus tout aussi teleos; que les Charites sensuelles sont présentes ainsi que les Saisons, les Hôrai; qu'Hermès est de la partie ainsi qu'Artémis. Il en va de même des " œuvres de guerre », lesquelles mobilisent Athéna et Arès, certes, mais aussi Enyô et Enyalios sans oublier le couple Arès et Aphrodite, dressé aux portes de la guerre, à Argos et peut-être en Crète. Et Zeus le sait aussi bien que Diomède, lui qui confirme à Aphrodite que, pour les œuvres de guerre, Athéna et l'ardent Arès y veilleront sans relâche. Ainsi que d'autres puissances dont le décompte n'est pas de circonstance : une Aphrodite d'Arès aurait été malvenue en face de Diomède et d'Athéna.

À suivre ce modèle indigène, fait à la maison - et laquelle ! celle d'Homère il y a, d'une part, des domaines respectifs assignés aux grands dieux, avec des limites; un dieu ne doit pas empiéter sur le domaine d'un autre; et gare au mortel qui oublie de reconnaître la compétence d'une puissance divine, s'il monte sur un navire, décide de se marier ou s'en va-t-en guerre. Par ailleurs, chacun de ces « grands domaines »-Dumézil disait «fonctions »- est traversé par une série de puissances, il est partagé entre des dieux souvent nombreux dont chacun semble prendre en charge un aspect, une dimension, une signification mi-

11 G. DUMÉzIL, L'béritage indo-européen à Rome, Paris, 1949, p. 78.

12 Iliade, V, 330-430. 
concrète, mi-abstraite. Le polythéisme est donc plus complexe que Zeus ne semble le dire sur l'Olympe ainsi que Dumézil, son grand écuyer, tranchant parmi les dieux à coups de modes d'action. Pour l'heure, notons que l'entreprise dumézilienne, et jusque dans les principes si féconds pour d'autres, se déployait dans le droit fil de la socio-anthropologie de Mauss et de l'impératif de découvrir des catégories et des classifications. Rien ne semblait alors plus neuf, plus stimulant que de considérer les " dieux en société » comme un vaste «système de classification »: Mitra et Varuna, enclos dans la souveraineté, offraient un principe formel de classification; les formules de la tripartition découvraient des ensembles articulés, des concepts, des manières de classer les grandes forces qui animent le monde et la société. Repérer des catégories dans les sociétés archaïques, recenser des formes de classification à l'œuvre dans les mythologies et les systèmes religieux, ce sont des entreprises qui n'ont pas cessé de servir au mieux la connaissance de l'esprit humain, considéré dans son histoire et dans ses variations.

Toutefois, en indiquant, à un certain moment de son enquête, que les dieux, plus que d'être définis par l'ensemble des positions qu'ils peuvent occuper, se délimitent par l'intention et les moyens de leur action, Georges Dumézil mettait les analystes des polythéismes en grand danger de renforcer le paradigme le plus traditionnel, celui du « dieu individué », le dieu que l'on peut identifier avec assurance et reconnaître à travers un certain nombre de traits constants. Ce que nous semblions gagner en faisant le relevé de l'ensemble des positions occupées par une divinité dans le système polythéiste en son entier, nous allions le perdre en dérivant vers un modèle de panthéon statique, habité par des dieux-agents, classés individuellement selon un mode d'action, unique et constant. En se laissant entraîner à définir un « dieu patron » par ses caractères fondamentaux et par son mode d'action en sa spécificité, Georges Dumézil nous ramenait à la case départ de l'analyse classique du panthéon. L'ouvrier patient et obstiné de la monographie en forme de thèse savourait déjà son triomphe : son choix était le bon, et, quant à la carrière, certes, il était et reste le meilleur eu égard au jugement de ses pairs historiens et de la confiance de son « patron », en cette vallée de larmes.

Il est temps d'en venir aux propositions d'expérimenter dans le champ des polythéismes, et, en particulier, dans ceux qui me sont le moins étrangers, dont les dieux de la Grèce, en premier. Voici vingt-cinq ans à peu près, j'ai essayé de cerner les différences entre des puissances divines qui semblaient avoir en commun un domaine d'activité, l'art hippique, la navigation ou la métallurgie, mais paraissaient y intervenir selon des modalités contrastées. Il s'agit d'une série d'analyses, directement inspirées des deux principes mis en avant par Georges Dumézil : que, pour définir une puissance divine, il faut entreprendre le relevé de l'ensemble des positions occupées par cette divinité dans le système polythéiste en sa totalité; qu'ensuite il convient de partir des groupements habituels et des associations cultuelles ou mythiques entre deux ou plusieurs puissances divines pour explorer la nature de leurs relations respectives, d'abord dans les contextes 
les plus explicites, et progressivement dans d'autres moins visibles et moins connus. Deux principes qui faisaient aussitôt réagir de manière critique les historiens de la religion grecque, garants institutionnels de la lecture monographique $^{13}$. Conduites dans le cadre d'une recherche sur les formes et les figures de l'intelligence rusée - menée alors avec la collaboration de J.-P. Vernant - ces analyses «structurelles », visant Athéna et Poseidon, Athéna et Héphaistos, tentaient de repérer des traits différentiels entre dieux conjoints dans une même forme d'intelligence pratique, mais selon des inflexions contrastées qu'il semblait judicieux de mettre en évidence sur l'horizon commun de la métis, cette « intelligence rusée » qui est également une puissance divine de rang cosmogonique, Métis, tôt gobée par le futur souverain de l'Olympe ${ }^{14}$. Il n'était pas inutile, je crois, d'observer de près des savoirs techniques communs à plusieurs dieux comme la métallurgie où se croisent Athéna, Héphaistos et les Telchines, ou bien, comme la navigation et « les manières d'user du cheval » qui mettent en scène au premier chef Athéna et Poseidon.

Sans résumer ces analyses, on peut en indiquer les limites dans la perspective « expérimentale » que nous entendons défendre aujourd'hui. En 1970-1974, il nous semblait éclairant de déployer à partir d'un culte commun à Poseidon et à Athéna, l'un dit Hippios, l'autre Hippia, la configuration de deux puissances «du cheval » : l'une chevaline, et l'autre, équestre tandis qu'elles semblaient se partager le terrain « hippique ». Deux grandes puissances se faisaient front dans une relation de complémentarité, fortement marquée à Corinthe, en particulier : Poseidon prenait en charge la violence, la fougue, la puissance inquiétante et incontrôlable de l'animal, tandis qu'Athéna se manifestait en agissant par le mors, par l'instrument technique de métal qui permet le maîtrise intelligente de l'animal et de sa force naturelle. L'analyse de 1970-1974 s'arrêtait sur les modes d'action respectifs de l'une et de l'autre puissances, en privilégiant dans le contexte «Ruses de l'intelligence » tout ce qui renforçait l'aspect «métis » d'Athéna, tantôt dite fille de Métis et de Zeus, tantôt identifiée elle-même à la plus haute métis des dieux. Or l'analyse, en procédant par recoupements entre récits et rituels et en découvrant plusieurs configurations voisines de la figure « corinthienne » initiale, invitait chemin faisant à conduire une série d'expériences qui auraient immédiatement rendu problématique le critère du mode d'action, constant et unique. Expériences simples comme, par exemple, prendre le cheval, analysé sous l'aspect «Athéna » et sous l'aspect «Poseidon »; le ou les mettre en contact « expérimental », d'abord, avec le dieu de la guerre, Arès, riche en chevaux et parfois en chevaux sacrifiés; ensuite, avec la divinité d'Argos, Héra, si désireuse de «pouvoir souverain » et volontiers belliqueuse, sinon franchement guerrière, car l'épouse de Zeus est ouvertement bippia. Autre mise en relation, cette fois davantage du côté de Poseidon : la Déméter d'Arcadie, la

13 C'est en leur nom que m'a été adressée la mise au point de F. ROBERT, Artémis et Atbéna, in Recuell Plassant, Paris, 1976, p. 135-157.

I4 M. DETIENNE, J.-P. VERNANT, Les ruses de l'intelligence. La métis des Grecs, Paris, 1974, (IV. Les savoirs divins : Athéna, Héphaistos), p. 169-260. 
noire, celle qui porte une tête de cheval, la Déméter Erinys et le cheval Arion, né de la saillie d'une Déméter cavale par l'étalon Poseidon. Il s'agit de voir quel aspect d'Arès mis en regard d'Athéna ou de Déméter affrontée à Poseidon peut soudainement faire découvrir une dimension inédite du cheval, qu'il soit attelé, monté ou sauvage, avec ou sans mors, dévorant ou inspiré. Et, réciproquement, ce serait l'occasion d'observer dans la configuration ainsi mise en place certaines dimensions d'Arès, d'Héra et de Déméter que d'autres manipulations permettraient de préciser, de corriger ou de rejeter, le cas échéant.

En se livrant à de telles expériences, l'analyste des dieux grecs se tient au plus près des données factuelles du champ polythéiste qui est le sien. Ce que nous appelons avec les Grecs le domaine du polutheos, de la pluralité des puissances divines se découvre à chaque page de la Description de la Grèce, écrite par Pausanias à l'époque d'Hadrien, mais aussi sur chaque pierre gravée révélant un calendrier de cité ou confirmant une association de divinités dans un sanctuaire, sur un autel ou dans l'organisation d'un rituel sacrificiel. Les groupements de dieux sont un aspect essentiel du paysage religieux de la Grèce que ce soit dans les hauts sanctuaires panhelléniques ou dans les villages perdus de l'arrière-pays. Ainsi, à l'intérieur du grand temple d'Apollon à Delphes, Pausanias a noté la présence d'un autel à Poseidon et d'autres témoignages précisent que deux autres divinités partageaient aussi le même espace : Gaia et Hestia ${ }^{15}$. Passons en Achaïe : près de Patras, dans la petite cité de Pharai, sur la place publique, un Hermès quadrangulaire et barbu se dresse à côté de l'autel d'Hestia, divinité du Feu public. En outre, dans ce cas, le couple Hermès-Hestia est mis en mouvement à travers un rituel divinatoire. Un peu plus loin, dans la même cité, Pausanias tombe sur un champ couvert de pierres : trente piliers quadrangulaires, anonymes et non sculptés. Or, en certains circonstances, les citoyens de Pharai se rendent en ce lieu jonché de pierres levées et « vénèrent les trente piliers en donnant à chacun le nom d'une divinité ${ }^{16}$. Allons vers Argos, cette fois en compagnie d'Eschyle. Arrivent les filles de Danaos pourchassées par leurs cousins depuis l'Égypte. Devant la cité, s'élève une petite colline, comme un sanctuaire rempli de dieux. Certains sont immédiatement identifiés par la troupe des Danaïdes : Zeus Hélios, le dieu Soleil, et Apollon. D'autres sont reconnaissables à des marques : le trident, c'est Poseidon; Hermès, le caducée. Ils ont des autels communs ${ }^{17}$. Comment sont-ils agencés? par triades, par couples, par pentades? Les combinaisons peuvent être variables, d'un lieu à l'autre, nous le vérifions très clairement dans le cas des « Douze dieux » : tantôt, six couples, à Olympie: tantôt, quatre autels de trois dieux, comme à Délos. Mais pourquoi pas deux fois six ou trois fois quatre? Passons au calendrier d'Erchia (dème attique) publié en $1963^{18}$. Col. A, 1. 44 et col. D, 1. 33, le même

PAUS., X, 24, 4 .

16 PAUS., VII, 22, 4 .

17 EsCH., Suppl., 189-222.

18 Cf. F. SOKOLowsKI, Lois sacrées des cités grecques, Paris, $1969, \mathrm{n}^{\circ} 18, \mathrm{p} .36-44$. 
jour du mois Élaphébolion : sacrifice à Sémélé; sacrifice à Dionysos. Une chèvre, un collège de femmes, la viande est partagée, la peau revient à la prêtresse, ou phora, à consommer sur place. Précision : "sur le même autel ». Autre association, en Gamélion, D, 1. 30 (avec B 37 et G 40) : sacrifice à Poseidon, même jour et même lieu que le sacrifice offert à Zeus Teleios et à Héra. Le lieu est le sanctuaire d'Héra à Erchia. Héra est en position d'hôte, d'une part. De l'autre, Poseidon est directement associé au couple protecteur du mariage. Comment et pourquoi ? Sur l'autel d'Amphiaraos, à douze stades d'Oropos, il y a dix-huit divinités sur une table d'autel, divisée en cinq parties. À Claros, en Asie Mineure, dans le grand sanctuaire oraculaire d'Apollon, l'autel principal est, cette fois, partagé entre Dionysos et Apollon.

Le polythéisme se lit en Grèce sur le sol, sur les autels, dans les temples, dans les règlements sacrificiels, dans les représentations figurées. Les matériaux que la culture grecque propose aux historiens de la religion, ce sont des agencements constitués, des relations organisées de deux ou de plusieurs puissances, des rapports d'opposition et de complémentarité explicites entre divinités. Le «structuré » est donc une donnée immédiate. Les panthéons grecs sont riches en groupements de dieux, en énoncés de hiérarchie, en figures de symétrie, d'antagonisme et d'affinité, aussi bien locaux que panhelléniques. En choisissant de travailler sur les groupements et les configurations de puissances divines, l'analyste des polythéismes grecs se montre résolument pragmatique, sinon même « positiviste ». Aux historiens de l'approche monographique qui classent paresseusement les «structures élémentaires» du panthéon sous la rubrique «associations » de tel dieu avec tel autre ainsi que fait depuis toujours l'Altertumswissenschaft ${ }^{19}$, il fera observer les innombrables récurrences d'associations et de groupements de divinités entre Homère et Porphyre, d'un bout à l'autre de l'Antiquité, depuis les confidences de Zeus sur les « domaines de compétence » des Olympiens jusqu'aux règlements cultuels, dédicaces et calendriers des cités grecques de la fin de l'époque hellénistique. Pendant dix siècles au moins, les Grecs ont les mêmes dieux, les mêmes sanctuaires, les même pratiques rituelles, ce qui n'exclut pas des changements locaux et des variations contextuelles. La longue durée du polythéisme grec offre à l'analyste un terrain d'expérimentation qu'il n'a pas encore, semble-t-il, exploité à la mesure des découvertes épigraphiques et des travaux d'érudition qui n'ont cessé d'enrichir et, souvent, de renouveler notre connaissance des panthéons et des pratiques cultuelles.

Depuis qu'il y a des historiens qui écrivent l'histoire de la pensée religieuse et l'histoire des dieux de la Grèce, la question de l'origine, sinon celle de l'étymologie, les fascine autant, sinon davantage, que le devenir, les vicissitudes d'une divinité allant d'un sanctuaire à l'autre, passant d'une configuration façonnée en tel lieu à la figure isolée et dominante que souhaitera lui donner

19 À travers une de ses productions majeures, par alleurs indispensable : Paulys Realencyclopädie der classischen Altertumswissenschaft. 
une cité à un moment de son histoire ${ }^{20}$. Selon F. Robert et d'autres, ce serait le devenir historique qui ferait les dieux, et sans doute, le reste. Je n'ai aucune raison décisive de récuser une enquête qui prétendrait trouver dans l'histoire et dans ses événements la clé d'un groupement de dieux ou d'un assemblage de puissances par un autel ou dans un sanctuaire. Peut-être pourrait-on objecter plus justement à l'historien en quête d'origine qu'il est contraint de fixer arbitrairement un premier contenu à la «personnalité » d'un dieu avant qu'elle ne commence à s'enrichir - et pourquoi pas s'appauvrir - au hasard de l'histoire et de ses accidents. Mais aucun analyste du polythéisme ne peut lire et reconnaître une configuration ou un simple couple de puissances sans avoir déjà une certaine idée de la silhouette ou de l'allure d'Athéna, de Poseidon ou d'Apollon. Toutefois s'il ne considère pas comme préjudiciel de penser qu'Athéna est, par exemple, «un paquet de fétiches ${ }^{21}$ (serpent, palladion, etc.), plus ou moins bien ficelé, ou encore qu'Apollon est sûrement un dieu hittite ou plutôt un asiate brutal et violent, l'analyste que je souhaite expérimental se sentira plus libre pour voir comment les groupements sont agencés, et surtout si les assemblages sont variables et diversifiés, ou encore pour analyser en profondeur la cohérence de certaines configurations autant que pour faire réagir, les uns par rapport aux autres, les dieux les plus contrastés.

Sur le terrain des polythéismes grecs, l'approche expérimentale se fera plus sûrement par des objets concrets, servant de « réactifs ${ }^{22}$, que par des mises en contact directes de puissances intégrales dont les traits individués, même implicitement, viendraient perturber les effets de la manifestation expérimentale. Le détour par les détails concrets et par les segments de situations est, sinon la voie la plus courte, du moins la plus sûre pour analyser les ensembles de relations entre dieux et ne pas se laisser séduire par les formes immédiates de dieux si portés à s'individualiser, pour leurs indigènes d'abord. Objets, gestes, situations: depuis les tracés entre dieux à métis, nous en avons évoqué quelques-uns : le mors, le cheval, le navire, le gouvernail; de même, du côté des gestes concrets, il y avait conduire, guider, cheminer, traverser, délimiter, ainsi que certaines situations dont la figure institutionnelle provisoire serait la guerre, le mariage, l'agriculture, la mort ou la naissance proliférant en gestes et en objets concrets, en principe illimités. Nous n'avons aucune peine à composer des listes du genre - dans un ordre alphabétique : arc, couteau, dauphin, fuseau, lance, phoque, rossignol, van, etc. Souvent même des érudits et des historiens dits des religions, curieux des « attributs » de certains dieux, en ont étudié, et très utilement, ce qu'ils appellent leurs symbolismes, rassemblant ainsi des morceaux d'encyclopédies et des conglomérats de représentations religieuses. Ce sont les

\footnotetext{
20 Perspective qui est celle de F. ROBERT, ant. cit. (n. 13).

21 Ainsi F. ROBERT, art. cit., p. 152.

22 Image suggérée par G. DuMÉzIL, Rituels indo-européens à Rome, Paris, 1954, p. 74.
} 
ethnologues, au premier rang desquels Lévi-Strauss ${ }^{23}$, qui ont mis en évidence la richesse des objets concrets, des gestes et des situations pratiques pour l'intelligence des récits mythiques et des aventures de personnages surnaturels. Ils nous font observer que tout objet, jouissant en principe d'un nombre de traits infini, peut être associé à d'autres objets dans des séries illimitées d'association. C'est ici qu'intervient la connaissance du contexte ethnographique qui doit permettre à l'analyste des ensembles polythéistes d'en savoir le plus possible sur la faune, la flore, les pratiques de jeux, de chasse, de guerre, sur tous les aspects matériels et concrets d'une culture. Qu'il s'agisse de lire des récits mythiques ou des configurations de dieux, l'helléniste, en l'occurrence, doit connâttre, non seulement les rituels, les calendriers, les règlements cultuels, mais aussi les écrits indigènes sur les plantes, les animaux, les pierres, les minéraux, les techniques, tout ce qui va lui permettre de « se brancher sur un réseau culturel ", plus ou moins aussi bien qu'un ethnologue en son village ou dans une ethnie dont les Observateurs de l'Homme auraient eu la patience d'écrire l'Encyclopédie ${ }^{24}$.

Les procédures expérimentales sont familières aux comparatistes qui entreprennent comme Lévi-Strauss de confronter deux systèmes différents, de prendre un objet facile à isoler, aux contours bien délimités et dont les différents états, révélés par l'observation, peuvent être analysés en recourant à quelques variables seulement dont tantôt on peut réduire le nombre, tantôt retenir celles qui sont d'un même type. De son côté, Dumézil, s'il a privilégié les concordances en plus d'une occasion, s'est aussi souvent exercé à voir, en marge d'une enquête sur la fonction guerrière (le Héros Troisième tuant un adversaire triple), comment chacune des sociétés mises en regard l'une ou l'autre imagine le destin du «Troisième Héros » après le meurtre commis, montrant ainsi qu'il y a au moins quatre façons de se débarrasser des suites d'une violence nécessaire ${ }^{25}$. C'est en prêtant attention aux articulations internes et aux détails significatifs que Dumézil a imaginé d'éprouver les incidences de la structure tripartie sur une série de «notions ou catégories de notions, concrètes et abstraites » qui semblent, à première vue, importantes, dans toute société ou, plus spécialement, dans les sociétés indo-européennes, son domaine de recherche ${ }^{26}$. Des objets, des gestes, des segments de situation : voilà donc les « réactifs », c'est-à-dire ce qui provoque une réaction au contact d'une puissance, d'un objet ou d'un geste qui va livrer un aspect inaperçu, une propriété cachée, un angle insolite. Expérimentation dont le principe le plus simple est de voir « ce qui se passe ».

23 Partout dans son cuvre depuis "La geste d'Asdiwal " (1958), mais très lumineusement dans son récent ouvrage : Histoire de Lynx, Paris, 1991, p. 249-255.

24 Méthode que nous avons pratiquée dans les Jardins d'Adonis, Paris, 1972, en y revenant plus attentivement dans la Postface "Où en sont les jardins d'Adonis?" (Paris, 1989², p. 243-263).

25 Cf. G. DUMÉzIL, Á propos des aspects de la fonction guerrière, in Cabiers pour l'Analyse, (Cercle d'épistémologie de l'E.N.S.) n 7, Paris, 1967.

26 Il faut rappeler ici combien cet aspect a été mis en évidence par un lecteur latiniste de Dumézil : J. SCHEID, G. Dumézil et la méthode expérimentale, in Opus (Rome), II (1983) p. 343-354. 
Ainsi que Dumézil nous l'a fait découvrir à propos du cheval ${ }^{27}$, qui n'est pas un animal pris au hasard dans une « civilisation » que la fonction guerrière, montée sur char, a entraîné vers de si foudroyantes conquêtes. Mis en contact avec un représentant qualifié de chacune des trois fonctions, le cheval fait voir trois aspects de sa nature animale en même temps qu'il accuse certains traits de la tripartition : attaché au quadrige du dieu souverain, le cheval fait saillir la vertu du triomphateur, s'avançant sous le signe de Jupiter dont le Flamine jamais ne peut monter ce noble animal, encore moins l'offrir en sacrifice; offert en sacrifice éclatant au dieu de la guerre, Mars en l'occurrence, le même animal exalte les vertus guerrières et les valeurs de la mort; présenté à la troisième fonction, le cheval se confond avec les équidés et devient une bête de somme, égale au mulet et à l'âne. Le monde indo-européen aurait-il connu cinq fonctions, le cheval se serait enrichi de deux autres qualités. De même l'objet mors qui semble absent du domaine indo-européen peut servir de réactif dans une configuration rassemblant en diverses formes d'association des divinités grecques, Athéna, Poseidon, les Telchines, Héphaistos et d'autres encore.

C'est en se montrant attentif au plus concret que le microanalyste se donne les moyens d'entrer expérimentalement dans les configurations de puissances divines qui sont les données primaires et les formes élémentaires proposées à l'observateur des polythéismes en pays grec. Prenons un dieu comme Apollon ${ }^{28}$ dont on sait combien, depuis l'fliade, il est impliqué dans de hautes configurations : dans ses épithètes, ses cultes, ses rituels, il offre à qui sait y prêter attention des manières très concrètes dont l'épopée homérique témoigne aussi directement que l'Hymne consacré à sa louange au $\mathrm{vI}^{\mathrm{e}}$ siècle avant notre ère. Partout en Grèce et pendant des siècles, Apollon est qualifié d'Aguieus : dieu des chemins, des routes, de la voirie. Il ouvre la route, il marque les chemins avec des pierres, par exemple, celles qui sont portées, véhiculées par ses prêtres de Milet au $\mathrm{vl}^{\mathrm{e}}$ siècle avant notre ère, dans la procession de dix-sept kilomètres reliant les portes du sanctuaire milésien aux portes du sanctuaire d'Apollon de Didymes. Donc, des pierres dressées le long des rues, des pierres portées, processionnées, en même temps qu'Apollon, lui-même, reçoit une présence figurative et cultuelle à travers un pilier. L'Hymne bomérique à Apollon raconte longuement comment le dieu, né à Délos, aménage l'espace, pose le pied, revient sur ses pas, fabrique du territoire, organise l'espace à traverse sa maîtrise de la voirie. Tout un vocabulaire concret attend l'interprète : mettre le pied sur, défricher-débroussailler, poser-fonder. Car Apollon commence par défricher la brousse, il balise son itinéraire à travers « la forêt primitive » (bylè qui signifiera plus tard la matière). Mieux, Apollon construit ses propres routes et, arrivé à Delphes, Apollon se met à poser des fondations de pierres, il fait se lever les

27 Cf. G. DUMÉZIL, Rituels indo-européens à Rome, Paris, 1954, p. 73-91; La religion romaine archaïque, Paris, 1966, p. 276-278.

28 Pour les références et les développements nécessaires, cf. Apollon. Le Bel Homicide de Delphes, à paraître (Paris, Gallimard). 
murs, il fait placer la pierre de seuil. Il est architecte et fondateur, au sens plein. Sur les chemins d'Apollon, qui croisent ceux d'Hermès et les carrefours d'Hécate ainsi que les sanctuaires de Poseidon, il y a des pierres portées, véhiculées, qu'il faut poser et fixer en certains lieux. Il y a aussi des seuils à poser, à franchir, à défendre; des portes à construire, à protéger; des enceintes à tracer, des limites à dessiner, à parcourir, ou à interdire.

Que fait un microanalyste devant ces pierres et ces portes? Il ne perd pas de temps à se demander si nous n'avons pas là les « preuves » du passage de l'ani conique à la figuration anthropomorphique. L'Apollon du Belvédère coexiste sans état d'âme avec un Apollon-pilier ou en forme de pierre conique comme est représenté le banal aguieus. Le microanalyste sait par la série des épithètes et le relevé des agencements que le dieu Hermès, le petit frère d'Apollon, partage avec le dieu de Délos, de Delphes ou de Mégare une série de qualités ouvrant sur les portes et sur les chemins, sur les seuils et sur les pierres, sur les enceintes et sur les autels, sans parler de la musique, de ses instruments ou encore de la parole et de ses effets. Voilà le champ d'expérimentation et de manipulations qui l'attend.

À ce stade de l'enquête, l'analyste qui n'a pas jugé nécessaire de se munir préalablement d'une fiche d'identité d'Apollon se trouve en possession d'un certain nombre de marques concrètes de ce dieu, et, à travers celles-ci, il entrevoit des orientations à l'intérieur d'un domaine plus ou moins apollinien, étant donné qu'il a déjà constaté que ce « domaine » recoupait le champ d'activité de plusieurs autres puissances, les unes régulièrement associés entre elles, les autres occasionnellement mises en relation. Le temps est venu, et c'est la seconde étape, de choisir une configuration parmi celles du champ apollinien et de chercher le sens du groupement ou les significations des articulations que l'analyste va essayer de reconnaître en les mettant à l'épreuve de tout ce qu'il peut savoir des polythéismes et de la société qui en use autant qu'elle en témoigne. La configuration du sanctuaire de Delphes, par exemple, rassemble autour d'Apollon Poseidon et Hestia, ainsi que Gaia à proximité de Thémis, puissances auxquelles il convient d'ajouter Dionysos qui n'est pas le dernier venu en ce milieu très choisi de l'oracle pythien. En règle générale, le microanalyste, placé devant un groupement de dieux, préfère un couple ou une triade plutôt qu'un assemblage aussi complexe que celui d'Apollon à Delphes. S'il s'agit d'un couple comme, par exemple, Hestia-Hermès, ou Apollon-Hermès, l'analyse peut se faire selon deux voies : d'une part, par exploration interne des significations de chacune des deux puissances en les mettant à l'épreuve de récits, de cultes, de rituels et d'institutions dont certains favorisent également l'action conjointe et contrastée des deux divinités qui semblent exprimer une structure du panthéon. L'autre voie, et elle est, me semble-t-il, complémentaire de la première, conduit à faire réagir la paire Hestia-Hermès ou Apollon-Hermès en les mettant en contact, et souvent en exploitant les relations explicites, avec l'ensemble de groupements disponibles, en commençant par les plus voisins comme Hestia-Apollon-Poseidon, Hermès-Aphrodite-Hestia, Poseidon-Gaia- 
Apollon-Thémis, par exemple. D'une manière ou d'une autre, l'approche reste expérimentale et vise à reconnaître des ensembles de relations sous-jacentes.

En l'occurrence, la configuration de l'Apollon de Delphes surgit sous les pas fondateurs d'un dieu appréhendé soigneusement à travers les objets, les gestes et les situations concrètes qui le font advenir entre Délos et Delphes. Pour aller en direction de Gaia, de Poseidon et d'Hestia, il est recommandé de suivre Apollon pas à pas, comme il est raconté, en l'occurrence, dans l'Hymne bomérique. Parallèlement au verbe ktizein qui conjoint le geste de débroussailler, d'ouvrir une voie à travers la forêt primitive, et l'acte de poser durablement, de fonder, l'action signifiée par bainein et ses composés indique un mouvement de passage d'un lieu à un autre, en mettant fermement «le pied sur ". Le pas d'Apollon fait trembler. Son pied est celui d'un buteur; il écrase; il imprime sa marque. Solidement planté sur ses jambes, le dieu protège villes et territoires : il amphibainei, fermement campé « de part et d'autre ». Une stabilité homologue à celle du chemin, bien construit, reliant un point à un autre, un chemin qui connaît un début et une fin, une archè et un télos. Cette stabilité apollinienne vient à la rencontre du seul dieu visible dans le parcours menant à Delphes. Il s'agit de Poseidon, et non pas de Gaia : le Poseidon d'Onchestos, régnant en son bois sacré sur le cheval nouvellement dompté et sur le char attelé. Poseidon est déjà là. À Delphes, il rattrape Apollon et lui offre « de purs soubassements »(dapeda), le sol aplani sur lequel le neveu, pleinement fondateur, pose les themeilia, la pierre de seuil, les murs porteurs, tout ce que exige un socle, une assise sûre, asphaleios, qui est une qualité éprouvée de Poseidon. Singulièrement, en des sites où il se présente en cofondateur, complice d'Apollon : lequel assume seul le rôle d'Archégète, laissant à l'oncle la vertu de themelioûchos, qui tient solidement les fondations.

Le couple formé par Apollon et Poseidon dans le temple de Delphes ainsi que la position liminaire d'un petit sanctuaire de $G \hat{e}$, invitent à ne pas partager le credo des archéologues delphiens pour qui une Terre d'évidence oraculaire se trouve en harmonie avec un site naturel à vocation mantique, offrant avec une source et des arbres la faille et le laurier. Comme l'on sait, après cent ans de fouilles au peigne fin, la $G \hat{e}$ de Delphes en son activité prophétique demeure introuvable. Muette, là, comme à Olympie, elle signifie un avant de par ses virtualités oraculaires qui se manifestent dans ses conseils adressés aux dieux en devenir. En marge du temple d'Apollon et à l'horizon de Delphes, Terre représente, certes, l'assise sûre à jamais, celle que sous-entend le Poseidon des «purs fondements ». Ce n'est pas la Gaia dont certains veulent en faire le «parèdre » (comme aime à dire l'histoire des religions en sa langue de bois), mais la Terre appelée la Bien Fondée, Eutbemetblos, parce qu'elle se fonde elle-même et en elle-même. En filigrane, une hiérarchie habite Delphes : l'Autofondée, le Poseur de socles et le Bâtisseur-Fondateur.

Hestia qui siège au cœur de la demeure apollinienne se réfère également à Terre par Rheia, son aïeule. Sa force de permanence et sa vertu de fixité se focalisent dans le foyer, le Foyer-autel de la maison, pour la Pythie, pour les 
sacrifiants consultants. Hestia, devenant Feu «pur et éternel » pour l'ensemble des cités grecques, Foyer Commun, mais se faisant puissance d'ouverture vers les cités nouvelles, leurs autels et leurs Prytanées. Hestia encore, en Dômatitis, active dans la construction de la maison, et courtisée chez elle par Apollon et par Poseidon, qui tous deux se qualifient et par leur épithète de dômatitès et par leur attirance pour la belle Fixité d'Hestia, pour son «assise immuable », si désirable pour l'oncle et pour le neveu. Lequel toutefois n'en attend rien pour la parole oraculaire qui laisse Hestia aussi muette que Poseidon.

Tandis que la nourrice du jeune dieu, Thémis, loin de patronner « les oracles de Terre », s'institue comme celle qui, intimement associée à Apollon, conduit vers les themistes, les «paroles fondées » ou les thesmol, les « décrets » que rend le Seigneur de Delphes lorsqu'il parle et themistenei. Médiatrice entre Gaia et Apollon, Thémis siège près du trépied oraculaire, en Pythie qui donnerait à la parole mantique ses valeurs les plus importantes. Mère à la fois des Moirai (les Parts qui sont deux et, à leur tête, l'Apollon Moiragetès) et des Hôrai (les Saisons qui règlent l'ordre social), Thémis se montre experte à dire ensemble en ses décrets le présent et le futur, donnant à la parole oraculaire sa puissance fondatrice depuis le sanctuaire divinatoire établi en premier et avant toute autre fondation, par Apollon, le nourri de Thémis. Autre entrée possible, par les sentiers de bainein : un Apollon " des bords de mer ", dieu « maritime » appelé tantôt Embasios, tantôt Ekbasios, embarquant ou débarquant, croisant ainsi Poseidon, Athéna ou Héra. Un Apollon que l'expédition des Argonautes permet de mettre en relation avec la consultation de l'oracle, l'autorisation du départ, un parcours aller-retour et des modalités d'intervention au plus près de celles que découvrent la fabrication du navire, la navigation et les périls de la mer du point de vue d'autres puissances plus ou moins « maritimes ».

En procédant de cette manière, l'analyste des configurations du polythéisme se donne la liberté de démonter et de remonter ce que Clifford Geertz appellerait " des logiques partielles de pensée ». Au lieu de choisir, initialement et souvent sans l'avouer, entre deux portraits-robots d'un dieu comme Apollon dieu de la supériorité morale (W.F. Otto) ou dieu terrible et violent (J. Defradas) - le microanalyste va fragmenter, il va focaliser sur des détails. Plus il va restreindre le champ de la comparaison - le pied et le seuil, par exemple entre Hermès et Apollon -, plus il va trouver de différences, de distinctions possibles entre deux puissances et d'autres convoquées pour l'occasion. Il est sûrement intéressant de faire réagir Poseidon, Hestia ou Héraclès devant un seuil, le mouvement d'un pied ou une pierre, qu'elle soit portée ou fichée en terre.

Autant de manières d'entrer dans les petits systèmes de pensée logés dans les assemblages et les groupements de dieux, et de voir comment, selon les suggestions de G. Lenclud, l'adoption d'un élément de pensée, d'une règle d'action invite une culture à faire des choix, quelles sont les contraintes à l'œuvre dans la configuration retenue, en fonction de son contexte culturel. Plus que d'expérimentations, il conviendrait de parler des manipulations, car si l'expérimentation exige aussi bien la répétition des phénomènes livrés à l'obser- 
vation que la possibilité d'intervenir sur les conditions de l'expérience, les manipulations peuvent couvrir ce que nous voulons dire en parlant de mettre en contact, de faire réagir les uns par rapport aux autres des phénomènes et des configurations qui ne sont jamais réitérés intégralement dans le cours de l'histoire mais dont une culture, interrogée dans la profondeur de sa durée, offre des récurrences formelles dans des contextes assez variés pour faire voir les transformations de certains des éléments composant ces phénomènes et ces configurations.

Nous voulons le dire à nouveau : l'approche expérimentale que nous proposons n'entend nullement méconnaître les traits qui donnent son style au polythéisme des Grecs : que les formes (schèmata) des Olympiens se découpent nettement sur les premières lueurs de la culture contemporaine d'Homère; que par la vertu des peintres et des sculpteurs les figures divines s'individualisent très vite et partout; que les poètes et les artisans du verbe n'ont cessé de parer les grands dieux de récits et de louanges strictement ajustés à chacun d'eux; ou encore, que, selon le temps et le lieu, les paysages du polythéisme se sont modifiés ou ont été redessinés ponctuellement selon un dessein arrêté. La liberté avec laquelle certaines assemblées politiques réorganisent le calendrier des fêtes et la hiérarchie des sacrifices est une donnée importante de la pratique polythéiste en Grèce. Ce faisant, les cités grecques ne livrent pas les agencements de dieux au hasard ni à l'arbitraire; elles expérimentent plutôt dans le quotidien certaines des combinaisons offertes par le système et ses potentialités.

En attirant l'attention sur tout ce qui n'est pas dit en clair des dieux et de leurs pouvoirs, nous voulons inviter les analystes des ensembles polythéistes à découvrir comment les puissances divines sont connectées par des dizaines de facettes à l'ensemble des objets et des phénomènes de la vie sociale et du monde naturel. Certes les dieux peuvent être mis en relation explicite dans des configurations dont certaines se déploient en récits tantôt mythologiques, tantôt «théologiques » au sens grec, mais ils sont d'abord pris dans la série des microréseaux qui les présentent en interaction complexe dans toute l'étendue du champ culturel. Seules des manipulations répétées permettent d'entrevoir progressivement la richesse du tissu polythéiste dans des sociétés où chaque dieu est d'abord au pluriel.

Marcel DETIENNE

Johns Hopkins University, BALTIMORE

École Pratique des Hautes Études, PARIS 